\title{
EFFECT OF AUDIOVISUAL ANXIETY CONTROL METHODS WITH AND WITHOUT NOISE CANCELATION FOR ADULT PATIENTS UNDERGOING ROUTINE DENTAL PROCEDURES "RANDOMIZED CONTROLLED CLINICAL TRIAL"
}

\author{
Hamad N. Albagieh*, Abdullah S. Alayad ${ }^{* *}$, Abdullah M. Aldosari***, Ahmed A. Alebdi***, \\ Sulaiman I. Alnajashi ${ }^{* * * *}$, Abdulwahab O. Aljardi*****, Sultan A. Alhazza******* and Emad M. Hadlaq*
}

\begin{abstract}
Objectives: the aim of the present study was to evaluate the effects of audio or audiovisual distraction with and without noise cancellation on the anxiety levels of patients undergoing routine dental procedures.

Material and Methods: A minimum random sample of 120 patients was selected on the basis of inclusion and exclusion criteria primarily pertaining to age (17-70 years), health (American Society of Anesthesiologists performance status I or II), and type of dental treatment (basic restorative procedures for class I, II, III, IV, and/or V lesions). The participants were divided into four main groups depending on the type of anxiety control method used during the dental procedure: control, audio distraction, audiovisual distraction, and noise cancellation. Different subgroups were created within each group on the basis of different method combinations. Anxiety levels were assessed by measuring the blood pressure, heart rate, and oxygen saturation levels before and after treatment. In addition, the State-Trait Anxiety Inventory (STAI) and Modified Dental Anxiety Scale (DAS) were also used to determine the effects of the anxiety control methods.
\end{abstract}

Results: Both audio distraction and audiovisual distraction significantly improved the STAI and DAS scores, regardless of the use of noise cancellation. Anxiety levels were significantly higher in the noise cancellation and control groups than in the audio distraction and audiovisual distraction groups.

Conclusion: Our findings suggest that audio and audiovisual distraction methods are effective in minimizing fear and anxiety during routine dental procedures in adult patients.

\footnotetext{
* Assistant Professor, Oral Medicine \& Diagnostic Sciences Department, College of Dentistry, King Saud University, Riyadh, Saudi Arabia

** Assistant Professor, Restorative Dental Sciences department, College of Dentistry, King Saud University, Riyadh, Saudi Arabia

*** Demonstrator, Prosthodontics Department, College of Dentistry, King Saud University, Riyadh, Saudi Arabia

**** General Dentist, Ministry of Health, Riyadh, Saudi Arabia

***** General Dentist, Smile Private Dental Clinic, Riyadh, Saudi Arabia

****** General Dentist, Saudi Security Forces, Riyadh, Saudi Arabia
} 


\section{INTRODUCTION}

One of the objectives of dental treatment is the promotion of optimum oral health, which has been shown to affect the general health of an individual. Brennan et al studied the correlation between oral health and general health and established that individuals with poor general health also exhibited oral health problems. ${ }^{(1)}$ Therefore, dental clinicians should use all necessary means to optimally treat their patients while causing minimum discomfort and anxiety. All efforts should be taken to increase the patient's compliance, which is undoubtedly affected by the degree of discomfort and anxiety during the treatment.

In recent years, dentists have used both pharmacological and nonpharmacological behavior management techniques to overcome dental anxiety, with pharmacological methods shown to be more effective, particularly in cases of severe anxiety. However, these methods not only require training but are also expensive and can result in side effects due to the medications used. ${ }^{(2)}$ As a result, clinicians have resorted to nonpharmacological behavior management techniques, which are more feasible and do not require complicated training. For example, De Jong et al used distraction and hypnosis for pain relief during the treatment of burn injuries. (3) In 1959, Gardner and Licklander introduced audio analgesia as a pain control method during dental treatment. ${ }^{(4)}$ Subsequently, many studies evaluated the usefulness of audio distraction during dental treatment, particularly in pediatric patients. (5-7) Other studies proved that music decreases the intensity of pain and the amount of anesthetic during treatment. However, the authors of these studies did not achieve the expected outcomes with regard to distraction and relaxation. ${ }^{(8-10)}$ Morse reported that audiovisual stimulation (AVS) can effectively minimize dental anxiety. ${ }^{(1)}$ MuViCure (Photobia ApS, Copenhagen, Denmark) was developed on the basis of the assumption that natural scenes and sounds in combination with music tend to minimize anxiety and stress. Nikolajsen studied the effects of MuViCure in patients receiving femoral nerve blocks and stated that it did not provide pain relief, stating that the environment of the clinic was a contributing variable that could not be controlled. ${ }^{(12)}$ Other authors have listed odors, windows, the interior design, and sunlight as factors that increase or decrease a patient's anxiety. ${ }^{(13,14)}$

On the basis of the above findings, we designed the present study to determine the effects of audio or audiovisual distraction with and without noise cancellation on the anxiety levels of adult patients undergoing routine dental procedures.

\section{PATIENTS AND METHODS}

\section{Patient grouping and dentist collaborations}

Five general dentists who completed their education and training at King Saud UniversityCollege of Dentistry were recruited for this crosssectional study. Before the start of the study, the four practitioners received a verbal explanation along with paper handouts about the study methodology in order to ensure a unified systematic approach for all patients.

In total, 132 patients aged 18 to 45 years who visited the dental clinics at King Saud University were randomly selected. All patients exhibited an American Society of Anesthesiologists performance status of I or II with no mental, visual, or hearing disabilities, and they required basic restorative procedures for class I, II, III, IV, and/or V lesions. They were divided into four main groups on the basis of the anxiety control method used during the treatment: control ( $\mathrm{n}=12$, group 1$)$, audio distraction $(\mathrm{n}=48)$, audiovisual distraction $(\mathrm{n}=48)$, and noise cancellation $(\mathrm{n}=24)$. The audio distraction group comprised four different subgroups ( $\mathrm{n}=12$ each) according to the additional use of noise and visual cancellation: audio distraction + noise and visual 
cancellation (group 2), audio distraction + noise cancellation (group 3), audio distraction + visual cancellation (group 4), and audio distraction only (group 5). Similarly, the visual distraction group comprised four subgroups ( $\mathrm{n}=12$ each): visual distraction only (group 6), visual distraction + noise cancellation (group 7), visual distraction + noise cancellation + audio distraction (group 8), and audiovisual distraction (group 9). The noise cancellation group comprised two subgroups according to the use of noise-cancelling headphones (group 10) or regular headphones (group 11) (Figure 1). In total, 11 groups were created and assigned using a randomization table according to which each of the four clinicians dealt with a different group of patients on each day.

\section{Preoperative consent and data collection}

Before study initiation, all patients were asked to read and sign an informed consent form that summarized the study procedures and informed them about their rights to discontinue the study at any point of time without bearing any negative effects on the quality of the treatment they were undergoing.

Before each procedure, the patient's anxiety levels were assessed by measurement of the blood pressure, heart rate, and oxygen saturation levels using a vital signs monitor (Mindray, China). Subsequently, each patient was asked to complete the 20 questions in the trait anxiety (T-anxiety) scale of the State-Trait Anxiety Inventory (STAI) in order to determine how he/she felt in general. (15)

\section{Procedures}

The anxiety control methods during the dental procedure varied among the different groups. In the control group, no anxiety control method was used to alter the treatment experience of the patient. In the audio distraction group, patients received noise-cancelling ( $n=24$; Bose QuietComfort 35 II, Massachusetts, United States) or regular $(n=24$;
Sony Wireless Stereo Headset 2.0; Tokyo; Japan) headphones playing Weightless by Marconi Union. For additional visual cancellation in the relevant subgroups, the patient was blindfolded for omission of surroundings. In the visual distraction group, patients were exposed to a virtual retinal display (Homido V2, Lille, France) playing different videos and were provided with noise-cancelling $(\mathrm{n}=24)$ or regular headphones $(n=24)$. For additional audio distraction in the relevant subgroups, the headphones provided to the patient played the same number used in the audio distraction group (Weightless by Marconi Union). In the noise cancellation group, patients received either noisecancelling headphones or regular headphones without any audio or audiovisual distraction.

All patients underwent basic restorative procedures for class I, II, III, IV, and/or V lesions, which involved injection of local anesthetic solution, rubber dam isolation, cavity preparation using high- and low-speed handpieces, restoration placement, and finishing and polishing of the final restoration. The duration of the appointment was no longer than $1 \mathrm{~h}$.

\section{Postoperative data collection}

Following completion of the dental procedures, the patients' anxiety levels were assessed again by measurement of the blood pressure, heart rate, and oxygen saturation levels. Subsequently, each patient was asked to complete the 20 questions in the state anxiety (S-anxiety) scale of STAI in order to determine how he/she felt after the treatment.(15)

\section{Statistical analysis}

The obtained data were tabulated and subjected to statistical analyses using one-way analysis of variance with post hoc analysis [least significant difference (LSD) test] and the paired samples $\mathrm{t}$-test. The level of significance was set at 0.05. All statistical analyses were performed using IBMSPSS version 25 (IBM Corp, Armonk, NY). 


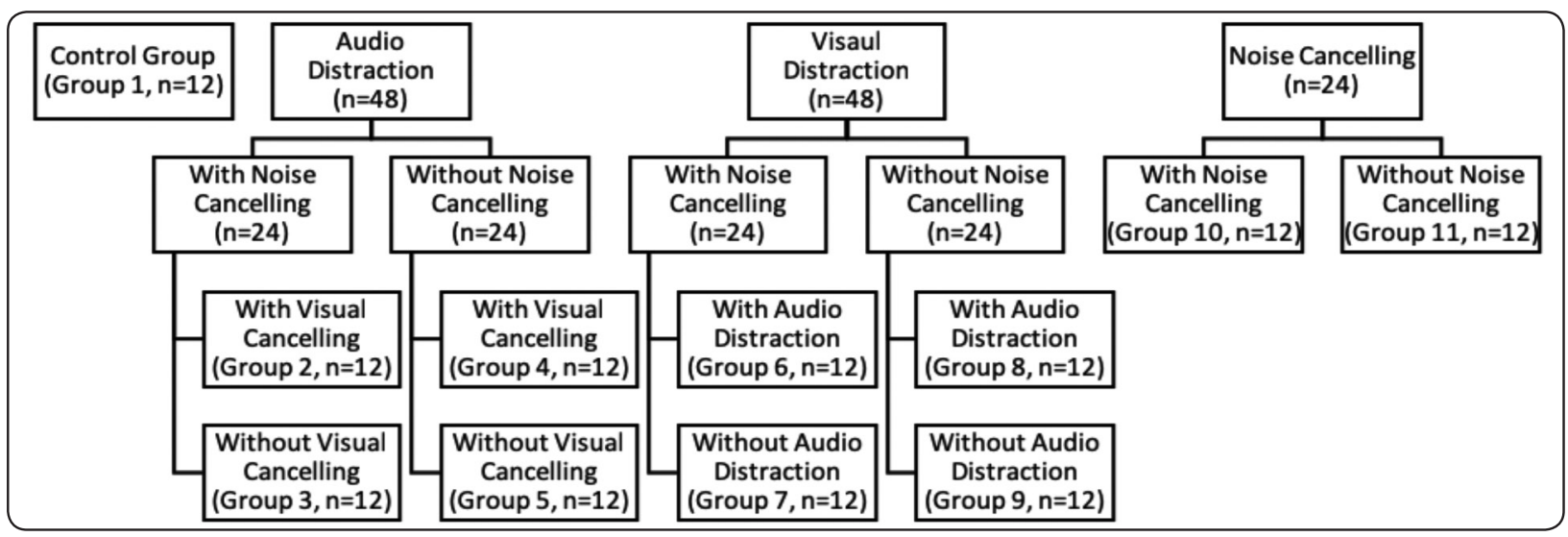

Fig. (1) Flowchart for a study on the effects of audiovisual methods with and without noise cancellation on dental anxiety in adult patients

\section{Ethics}

This study was approved by the King Saud University College of Dentistry Research

Center (Registration number: IR 0210). The study protocol was designed in accordance with the 2002 revision of the Declaration of Helsinki, and all patients provided written informed consent for study participation.

\section{RESULTS}

Table 1 shows the mean STAI scores for the 11 groups. It was observed that the average degree of anxiety varied according to the anxiety control method. The methods used in groups 2, 3, 4, 5, and 6 had a greater influence on the scores than did the methods used in the other groups, including the control group (Graph 1). ANOVA revealed statistically significant differences in the mean S-anxiety scores between the control group and the other groups (Table 2), with the findings indicating that all anxiety control methods were effective in statistical terms. Post hoc analysis with the LSD test was used to evaluate the difference in the mean S-anxiety score between the control group and each test group. The results revealed that the methods used in groups $2-8$ resulted in significant decreases in the anxiety levels of patients, whereas those used in groups 9-11 were less effective (Table 3).
TABLE (1) The State-Trait Anxiety scores of the subjects in all groups

\begin{tabular}{|c|c|c|c|c|}
\hline$\underset{n}{\frac{\pi}{5}}$ & $\begin{array}{l}\text { ज्ञ } \\
\text { जे }\end{array}$ & 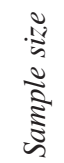 & $\frac{\tilde{\Xi}}{\Sigma}$ & ఉ \\
\hline \multirow{12}{*}{ 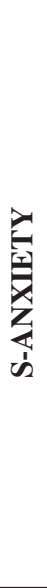 } & 1 & 12 & 2.9125 & .25238 \\
\hline & 2 & 12 & 2.5208 & .19360 \\
\hline & 3 & 12 & 2.5292 & .10326 \\
\hline & 4 & 12 & 2.7250 & .18028 \\
\hline & 5 & 12 & 2.5958 & .16301 \\
\hline & 6 & 12 & 2.4792 & .17769 \\
\hline & 7 & 12 & 2.6542 & .14687 \\
\hline & 8 & 12 & 2.7542 & .18273 \\
\hline & 9 & 12 & 2.8833 & .13707 \\
\hline & 10 & 12 & 2.8833 & .13707 \\
\hline & 11 & 12 & 2.7833 & .17753 \\
\hline & Total & 132 & 2.7019 & .22347 \\
\hline \multirow{12}{*}{ 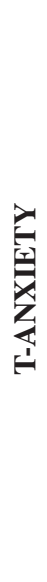 } & 1 & 12 & 3.1167 & .31358 \\
\hline & 2 & 12 & 2.8625 & .29165 \\
\hline & 3 & 12 & 2.9625 & .14943 \\
\hline & 4 & 12 & 2.9667 & .12309 \\
\hline & 5 & 12 & 2.8958 & .22709 \\
\hline & 6 & 12 & 2.9833 & .22496 \\
\hline & 7 & 12 & 2.9833 & .22496 \\
\hline & 8 & 12 & 2.9625 & .24414 \\
\hline & 9 & 12 & 3.0000 & 20780 \\
\hline & 10 & 12 & 3.0000 & .20780 \\
\hline & 11 & 12 & 3.0333 & .07487 \\
\hline & Total & 132 & 2.9788 & .21940 \\
\hline
\end{tabular}




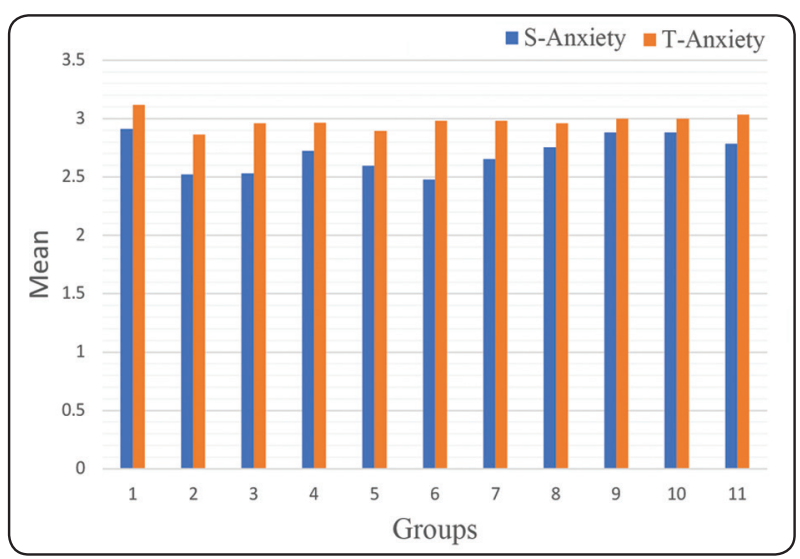

Graph 1. Mean State-Trait Anxiety Inventory scores for adult patients who underwent routine dental procedures with different audiovisual anxiety control methods

* Group1: Control, Group 2: Audio Distraction + Noise And Visual Cancellation , Group 3: Audio Distraction + Noise Cancellation, Group 4: Audio Distraction + Visual Cancellation, Group 5: Audio Distraction Only, Group 6: Visual Distraction Only, Group 7: Visual Distraction + Noise Cancellation, Group 8: Visual Distraction + Noise Cancellation + Audio Distraction, Group 9: Audiovisual Distraction, Group 10: Noise-Cancelling Headphones, And Group 11: Regular Headphones

TABLE (2) Findings of analysis of variance for mean State-Trait Anxiety Inventory scores for adult patients who underwent routine dental procedures with different audiovisual anxiety control methods

\begin{tabular}{|c|c|c|c|c|c|c|}
\cline { 2 - 7 } \multicolumn{2}{c|}{} & $\begin{array}{c}\text { Sum of } \\
\text { squares }\end{array}$ & $d f$ & $\begin{array}{c}\text { Mean } \\
\text { square }\end{array}$ & $F$ & P-VALUE \\
\hline \multirow{2}{*}{$\begin{array}{c}\text { Between } \\
\text { groups }\end{array}$} & 2.950 & 10 & .295 & 9.938 & .000 \\
\cline { 2 - 7 } & $\begin{array}{c}\text { Within } \\
\text { groups }\end{array}$ & 3.592 & 121 & .030 & & \\
\cline { 2 - 7 } & Total & 6.542 & 131 & & & \\
\hline \multirow{2}{*}{$\begin{array}{c}\text { Between } \\
\text { groups }\end{array}$} & .528 & 10 & .053 & 1.106 & .363 \\
\cline { 2 - 7 } & $\begin{array}{c}\text { Within } \\
\text { groups }\end{array}$ & 5.778 & 121 & .048 & & \\
\cline { 2 - 7 } & Total & 6.306 & 131 & & & \\
\hline
\end{tabular}

TABLE (3) Effectiveness of different audiovisual anxiety control methods used during routine dental procedures in adults in terms of the S-anxiety scale scores after treatment

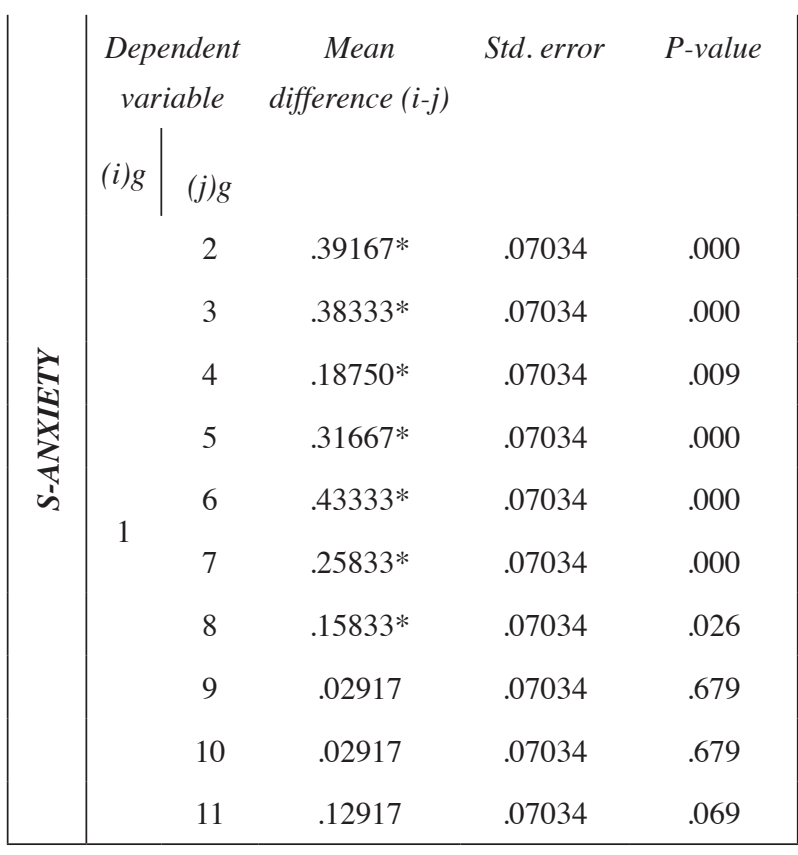

* Group1: Control, Group 2: Audio Distraction + Noise And Visual Cancellation, Group 3: Audio Distraction + Noise Cancellation , Group 4: Audio Distraction + Visual Cancellation, Group 5: Audio Distraction Only, Group 6: Visual Distraction Only, Group 7: Visual Distraction + Noise Cancellation, Group 8: Visual Distraction + Noise Cancellation + Audio Distraction, Group 9: Audiovisual Distraction, Group 10: Noise-Cancelling Headphones, And Group 11: Regular Headphones. SD: Standard Deviation.

Table 4 shows the effects of each anxiety control method on the blood pressure and pulse rate, as assessed using the paired samples t-test. Our analysis revealed that the methods used in groups 2, 3 , and 4 were the most effective in relieving anxiety by causing significant decreases in both the blood pressure and pulse rate.

Finally, Table 5 summarizes the overall effects of the anxiety control methods on both the questionnaire scores and physiological parameters. Our analysis revealed that the methods used in groups 2-8 significantly reduced the anxiety levels 
of patients, whereas those used in groups 9-11 were less effective (Graph 2).

TABLE (4) Findings of t-tests for evaluating the effects of different audiovisual anxiety control methods used during routine dental procedures on the blood pressure and pulse rate of adult patients

\begin{tabular}{|c|c|c|c|c|c|c|}
\hline 今ి & $\begin{array}{c}\text { Physiological } \\
\text { test }\end{array}$ & Mean & $N$ & $S D$ & $t$ & p-value \\
\hline \multirow{6}{*}{1} & Pre BPSYS & 132.08 & 12 & 13.173 & \multirow{2}{*}{0.651} & \multirow{2}{*}{.528} \\
\hline & Post BP SYS & 130.08 & 12 & 18.123 & & \\
\hline & Pre BP DIA & 74.33 & 12 & 14.336 & \multirow{2}{*}{-0.578} & \multirow{2}{*}{.575} \\
\hline & Post BP DIA & 77.25 & 12 & 13.383 & & \\
\hline & Pre PR & 80.00 & 12 & 10.938 & \multirow{2}{*}{.080} & \multirow{2}{*}{.938} \\
\hline & Post PR & 79.83 & 12 & 10.701 & & \\
\hline \multirow{6}{*}{2} & Pre BP SYS & 143.58 & 12 & 8.372 & \multirow{2}{*}{2.290} & \multirow{2}{*}{.043} \\
\hline & Post BP SYS & 136.75 & 12 & 7.759 & & \\
\hline & Pre BP DIA & 86.00 & 12 & 5.939 & \multirow{2}{*}{3.493} & \multirow{2}{*}{.005} \\
\hline & Post BP DIA & 76.67 & 12 & 4.793 & & \\
\hline & Pre PR & 83.42 & 12 & 4.963 & \multirow{2}{*}{6.504} & \multirow{2}{*}{.000} \\
\hline & Post PR & 75.08 & 12 & 3.630 & & \\
\hline \multirow{6}{*}{3} & Pre BP SYS & 145.42 & 12 & 10.140 & \multirow{2}{*}{5.631} & \multirow{2}{*}{.000} \\
\hline & Post BP SYS & 133.75 & 12 & 7.806 & & \\
\hline & Pre BP DIA & 82.33 & 12 & 12.419 & \multirow{2}{*}{4.471} & \multirow{2}{*}{.001} \\
\hline & Post BP DIA & 73.25 & 12 & 10.297 & & \\
\hline & Pre PR & 90.83 & 12 & 6.013 & \multirow{2}{*}{6.058} & \multirow{2}{*}{.000} \\
\hline & Post PR & 79.00 & 12 & 4.200 & & \\
\hline \multirow{6}{*}{4} & Pre BP SYS & 142.67 & 12 & 5.914 & \multirow{2}{*}{6.169} & \multirow{2}{*}{.000} \\
\hline & Post BP SYS & 128.33 & 12 & 7.190 & & \\
\hline & Pre BP DIA & 76.67 & 12 & 10.739 & \multirow{2}{*}{-0.7549} & \multirow{2}{*}{.466} \\
\hline & Post BP DIA & 78.58 & 12 & 13.235 & & \\
\hline & Pre PR & 89.17 & 12 & 3.407 & \multirow{2}{*}{6.146} & \multirow{2}{*}{.000} \\
\hline & Post PR & 79.58 & 12 & 5.501 & & \\
\hline
\end{tabular}

\begin{tabular}{|c|c|c|c|c|c|c|}
\hline \multirow{6}{*}{5} & Pre BPSYS & 142.58 & 12 & 13.104 & \multirow{2}{*}{4.129} & \multirow{2}{*}{.002} \\
\hline & Post BP SYS & 128.33 & 12 & 14.009 & & \\
\hline & Pre BPDIA & 80.67 & 12 & 6.853 & \multirow{2}{*}{.976} & \multirow{2}{*}{.350} \\
\hline & Post BP DIA & 77.42 & 12 & 10.698 & & \\
\hline & Pre PR & 85.67 & 12 & 4.658 & \multirow{2}{*}{3.963} & \multirow{2}{*}{.002} \\
\hline & Post PR & 79.42 & 12 & 4.420 & & \\
\hline \multirow{6}{*}{6} & Pre BP SYS & 127.92 & 13 & 9.403 & \multirow{2}{*}{2.510} & \multirow{2}{*}{.027} \\
\hline & Post BP SYS & 123.08 & 13 & 7.216 & & \\
\hline & Pre BPDIA & 77.08 & 13 & 3.968 & \multirow{2}{*}{1.647} & \multirow{2}{*}{.126} \\
\hline & Post BP DIA & 71.85 & 13 & 9.146 & & \\
\hline & Pre PR & 80.15 & 13 & 22.898 & \multirow{2}{*}{-0.585} & \multirow{2}{*}{.570} \\
\hline & Post PR & 83.31 & 13 & 5.851 & & \\
\hline \multirow{6}{*}{7} & Pre BPSYS & 132.25 & 12 & 14.322 & \multirow{2}{*}{-0.395} & \multirow{2}{*}{.700} \\
\hline & Post BP SYS & 133.67 & 12 & 10.307 & & \\
\hline & Pre BPDIA & 76.75 & 12 & 9.564 & \multirow{2}{*}{-1.88} & \multirow{2}{*}{.087} \\
\hline & Post BP DIA & 81.00 & 12 & 10.357 & & \\
\hline & Pre PR & 80.00 & 12 & 10.938 & \multirow{2}{*}{.761} & \multirow{2}{*}{.463} \\
\hline & Post PR & 79.50 & 12 & 9.765 & & \\
\hline \multirow{6}{*}{8} & Pre BPSYS & 140.75 & 12 & 7.569 & \multirow{2}{*}{3.613} & \multirow{2}{*}{.004} \\
\hline & Post BP SYS & 126.00 & 12 & 14.845 & & \\
\hline & Pre BPDIA & 82.33 & 12 & 14.883 & & \\
\hline & Post BP DIA & 81.67 & 12 & 10.782 & $.1 / 8$ & .802 \\
\hline & Pre PR & 83.42 & 12 & 4.963 & 7755 & 107 \\
\hline & Post PR & 80.67 & 12 & 3.774 & 1.155 & .107 \\
\hline & Pre BPSYS & 137.64 & 12 & 12.274 & & \\
\hline & Post BP SYS & 138.91 & 12 & 17.728 & $-0.2 \mathrm{~J}$ & .019 \\
\hline 9 & Pre BP DIA & 75.09 & 12 & 8.093 & & \\
\hline & Post BP DIA & 78.64 & 12 & 8.903 & -1.529 & $.21 J$ \\
\hline & Pre PR & 86.18 & 12 & 6.897 & 1780 & 104 \\
\hline & Post PR & 85.09 & 12 & 6.123 & 1.189 & .104 \\
\hline & Pre BP SYS & 137.17 & 12 & 11.816 & 040 & 060 \\
\hline & Post BP SYS & 136.92 & 12 & 18.258 & & \\
\hline 10 & Pre BP DIA & 75.50 & 12 & 7.845 & & \\
\hline & Post BP DIA & 78.83 & 12 & 8.516 & -1.504 & .200 \\
\hline & Pre PR & 77.17 & 12 & 11.320 & 20 & 070 \\
\hline & Post PR & 77.25 & 12 & 11.234 & -0.038 & .970 \\
\hline
\end{tabular}




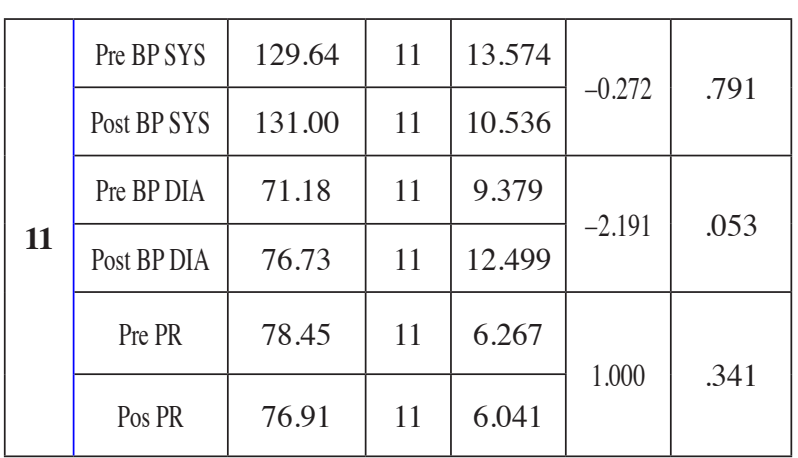

* Group1: Control, Group 2: Audio Distraction + Noise And Visual Cancellation, Group 3: Audio Distraction + Noise Cancellation, Group 4: Audio Distraction + Visual Cancellation, Group 5: Audio Distraction Only, Group 6: Visual Distraction Only, Group 7: Visual Distraction + Noise Cancellation, Group 8: Visual Distraction + Noise Cancellation + Audio Distraction, Group 9: Audiovisual Distraction, Group 10: Noise-Cancelling Headphones, And Group 11: Regular Headphones. SD: Standard Deviation, Pre BP SYS: Preoperative Systolic Blood Pressure, Post BP SYS: Postoperative Systolic Blood Pressure, Pre BP DIA: Preoperative Diastolic Blood Pressure, Post BP DIA: Postoperative Diastolic Blood Pressure, Pre PR: Preoperative Pulse Rate, Post PR: Postoperative Pulse Rate.

Table (5) Overall effects of different anxiety control methods used during routine dental procedures in adults in terms of S-anxiety scale scores and physiological parameters, including blood pressure and pulse rate

\begin{tabular}{|c|c|c|c|c|c|c|c|c|c|c|c|}
\hline Group & 1 & 2 & 3 & 4 & 5 & 6 & 7 & 8 & 9 & 10 & 11 \\
\hline S-Anxiety Scores & control & S & S & S & S & S & S & S & - & - & - \\
\hline $\begin{array}{c}\text { physiological } \\
\text { parameters }\end{array}$ & control & S & S & S & - & - & - & - & - & - & - \\
\hline
\end{tabular}

*s: statistically significant decrease, S-ANXIETY: state anxiety, * Group1: Control, Group 2: Audio Distraction + Noise And Visual Cancellation, Group 3: Audio Distraction + Noise Cancellation , Group 4: Audio Distraction + Visual Cancellation, Group 5: Audio Distraction Only, Group 6: Visual Distraction Only, Group 7: Visual Distraction + Noise Cancellation, Group 8: Visual Distraction + Noise Cancellation + Audio Distraction, Group 9: Audiovisual Distraction, Group 10: Noise-Cancelling Headphones, And Group 11: Regular Headphones

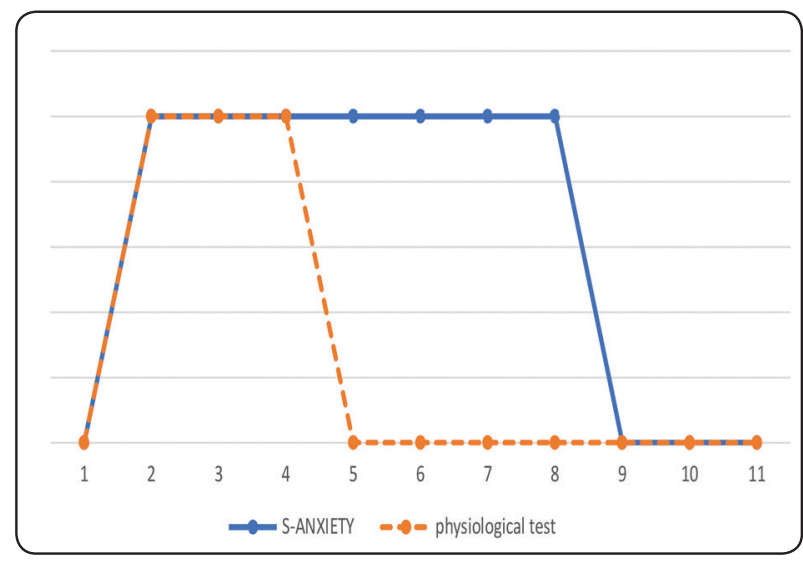

Graph 2. Overall effects of different anxiety control methods used during routine dental procedures in adults in terms of S-anxiety scale scores and physiological parameters, including blood pressure and pulse rate

* S-anxiety: state anxiety, * Group1: Control, Group 2: Audio Distraction + Noise And Visual Cancellation , Group 3: Audio Distraction + Noise Cancellation, Group 4: Audio Distraction + Visual Cancellation, Group 5: Audio Distraction Only, Group 6: Visual Distraction Only, Group 7: Visual Distraction + Noise Cancellation, Group 8: Visual Distraction + Noise Cancellation + Audio Distraction, Group 9: Audiovisual Distraction, Group 10: Noise-Cancelling Headphones, And Group 11: Regular Headphones

\section{DISCUSSION}

In the present study, we evaluated the role of audio and/or visual distraction with and without noise cancellation in the management of anxiety in adult patients undergoing routine dental procedures. Both audio and visual distraction significantly reduced the anxiety levels of patients, with audio distraction (groups 2-5) having a greater impact than audiovisual distraction (groups 6-9). Furthermore, noise cancellation alone (group 10) had no effect on the stress levels of patients, although it had a positive influence when used along with audio or audiovisual distraction.

Fear and stress are major problems affecting patients seeking dental care, and they can adversely influence the ability of practitioners to provide adequate help. In severe cases, they can drastically alter the treatment options or outcomes. Anxiety 
is mostly caused by a number of factors such as previous traumatic experiences, most likely during childhood, and uneasiness with regard to the sounds and appearances of dental instruments. ${ }^{(2)}$

Several behavioral and pharmacological methods have been implemented for dealing with anxiety, mainly in pediatric patients. On the other hand, few techniques for managing apprehensive adults in the dental clinic have been reported; as a result, general dentists have no specific means for handling such cases, creating a better dental experience for all involved parties, and achieving a pleasant outcome. ${ }^{(2)}$

In the present study, two methods were used for the assessment of dental anxiety in adult patients. The first involved the use of STAI, which comprises two parts designed to measure S-anxiety and T-anxiety and is the most commonly used method with proven validity and reliability. ${ }^{(17)}$ The second involved measurement of the patient's blood pressure and heart rate, which have been shown to be the most reliable indicators of stress and anxiety. $(18-21)$

A number of studies have concluded that audio distraction is an effective method for managing anxious pediatric patients during various treatment phases in the dental clinic. $^{(22-25)}$ According to a meta-analysis conducted by Song et al., music can reduce the pain and anxiety levels of patients undergoing biopsy. ${ }^{(26)}$ Rezvan studied the effects of the environment on patients undergoing urodynamic studies and concluded that adjustment of the lighting and playing of relaxing audio tunes were effective in managing the subjects with regard to stress and embarrassment. ${ }^{(27)}$ The findings of the present study confirm the positive influence of audio and audiovisual distraction, regardless of the use of noise cancellation, on the patient's experience during routine dental treatment, and this positive influence is expected to promote patient compliance and commitment to scheduled appointments.
As mentioned above, the use of nonpharmacological distraction methods for behavior control in the field of pediatric dentistry is well documented. A meta-analysis by Zhang et al. suggested that audiovisual distraction effectively reduced dental anxiety and could be used during dental treatment in children. ${ }^{(28)}$ In addition, a meta-analysis by Hudson et al. concluded that interventions using both relaxationbased and audiovisual distraction were helpful in reducing anxiety levels and relieving pain during surgeries under local anesthesia. ${ }^{(5)}$ In the present study, audio and/or visual distraction using VRD (groups 6 -9) effectively lowered the anxiety levels of adult patients, particularly when noise cancellation was additionally used (group 6 ); this combination was the most effective in reducing anxiety levels. These findings suggest that alteration of the patient's environment in order to create a more pleasant treatment experience is largely beneficial for both the patient and dentist.

As observed with any treatment modality, dental treatment is also associated with some difficulties and limitations that need to be overcome or compensated by modifications in order to achieve the desired outcome. In the present study as well, some problems associated with the anxiety control methods were observed by either the patient or the clinicians. First, visualization of the maxillary teeth, particularly in the anterior segment, was impaired by VRD, necessitating adjustment of the patient in a sitting position or adjustment of the clinician's position for greater accessibility. Second, disinfection of equipment between appointments requires some time; therefore, multiple sets that are ready for use should be available. Third, patientdentist communication is difficult with noisecancelling headphones, and the audio may have to be paused or the headphones may need to be removed for appropriate communication. Fourth, each patient may have different preferences regarding the audio and video, which becomes a challenge for the dentist. This problem can be resolved by asking the 
patient to connect their own device to the headset or VRD, if possible. Finally, some technical issues that can interrupt the flow of the procedure and are beyond the control of the dentist or nurse may occur. Accordingly, dentists and nurses should be adequately trained to promptly handle these issues and minimize the procedural time.

As do all scientific research; this study has some limitations. The sample was conveniently gathered form patients treated at King Saud University Dental Hospital. This confined sample may not represent all types of patients, especially those seeking care at private clinics. Five clinicians with the same educational background were recruited for this study, all underwent detailed explanation of the protocols of the research. Regardless of these facts each practitioner has his own unique style in communicating with his patients and how he conducts his treatment, these factors may have either positive or negative impact on the stress and anxiety levels of the patients. However, the study's findings support and are in agreement with a considerable amount of the previously published literature in the field. The well-trained nurses, high quality of equipment, pristine reputation of King Saud University - Dental Hospital, comfortable dental chairs, and the modern interior design of the hospital may contribute in the overall patient's anxiety, which is not present in other governmental or private dental clinics. In this study only patients undergoing operative care were evaluated, other more demanding dental procedures might cause greater anxiety, and implementation of audio and/ or visual distraction measures might be challenging in such environments. Which may require further scientific investigation to determine the best possible protocols for anxiety control. Everyday different manufactures are competing to produce new innovations in the field of audio and visual technology, that may provide greater relaxation and help in the management of patient anxiety by overcoming the limitation of the previous models.
The authors of this study encourage further research in the area of nonpharmacological management of adult patient's anxiety undergoing dental care, in order to implement better protocols and provide a pleasant environment for both patients and dental staff involved, that will enhance the treatment outcome.

\section{CONCLUSIONS}

In conclusion, the findings of this study suggest that audio and audiovisual distraction methods are effectiveinreducing the anxietylevelsofadultpatients undergoing routine dental procedures, regardless of the use of noise cancellation. These methods positively complement the clinical environment and provide a satisfying treatment experience for both the dentist and patient, thus influencing the patient's commitment and compliance during and after treatment. Further studies on methods for reducing dental anxiety in adult patients and providing a stress-free environment for the patient and clinician are necessary. The aim of future research should be to investigate the impact of different VRDs available in the market or develop new devices that are suitable for use by dentists. In addition, suppliers should take some efforts to minimize the sounds produced by dental instruments without compromising their effectiveness in order to provide a better treatment experience for all parties involved in dental treatment.

\section{REFERENCES}

1. Brennan DS, Teusner DN. Oral health impacts on selfrated general and oral health in a cross-sectional study of working age adults. Community Dentistry and Oral Epidemiology. 2015;43(3):282-8.

2. Gazal G, Tola AW, Fareed WM, Alnazzawi AA, Zafar MS. A randomized control trial comparing the visual and verbal communication methods for reducing fear and anxiety during tooth extraction. The Saudi Dental Journal. 2016;28(2):80-5. 
3. de Jong AE, Middelkoop E, Faber AW, Van Loey NE. Non-pharmacological nursing interventions for procedural pain relief in adults with burns: a systematic literature review. Burns : journal of the International Society for Burn Injuries. 2007;33(7):811-27.

4. Gardner WJ, Licklider JCR. Auditory analgesia in dental operations. The Journal of the American Dental Association. 1959;59(6):1144-9.

5. Hudson BF, Ogden J. Exploring the Impact of Intraoperative Interventions for Pain and Anxiety Management During Local Anesthetic Surgery-A Systematic Review and Meta-Analysis. Journal of perianesthesia nursing : official journal of the American Society of PeriAnesthesia Nurses. 2016;31(2):118-33.

6. Lahmann C, Schoen R,Henningsen P, Ronel J, Muehlbacher $\mathrm{M}$, Loew $\mathrm{T}$, et al. Brief relaxation versus music distraction in the treatment of dental anxiety: a randomized controlled clinical trial. Journal of the American Dental Association (1939). 2008;139(3):317-24.

7. Singh D, Samadi F, Jaiswal J, Tripathi AM. Stress Reduction through Audio Distraction in Anxious Pediatric Dental Patients: An Adjunctive Clinical Study. International Journal of Clinical Pediatric Dentistry. 2014;7(3):149-52.

8. Nilsson U. The anxiety- and pain-reducing effects of music interventions: a systematic review. AORN journal. 2008;87(4):780-807.

9. Shertzer KE, Keck JF. Music and the PACU environment. Journal of perianesthesia nursing : official journal of the American Society of PeriAnesthesia Nurses. 2001;16(2):90-102.

10. Cepeda MS, Carr DB, Lau J, Alvarez H. Music for pain relief. The Cochrane database of systematic reviews. 2006(2):Cd004843.

11. Morse DR. Brain wave synchronizers: A review of their stress reduction effects and clinical studies assessed by questionnaire, galvanic skin resistance, pulse rate, saliva, and electroencephalograph. Stress Medicine. 1993;9(2):111-26.

12. Nikolajsen L, Lyndgaard K, Schriver NB, Moller JF. Does audiovisual stimulation with music and nature sights (MuViCure) reduce pain and discomfort during placement of a femoral nerve block? Journal of perianesthesia nursing : official journal of the American Society of PeriAnesthesia Nurses. 2009;24(1):14-8.
13. Dijkstra K, Pieterse M, Pruyn A. Physical environmental stimuli that turn healthcare facilities into healing environments through psychologically mediated effects: systematic review. Journal of advanced nursing. 2006;56(2):166-81.

14. Malenbaum S, Keefe FJ, Williams AC, Ulrich R, Somers TJ. Pain in its environmental context: implications for designing environments to enhance pain control. Pain. 2008;134(3):241-4.

15. Spielberger CD GR, Lushene R, Vagg PR, Jacobs GA. Manual for the state-trait anxiety inventory, STAI (form Y) Mind Garden: Palo Alto. 1983.

16. Humphris GM, Morrison T, Lindsay SJ. The Modified Dental Anxiety Scale: validation and United Kingdom norms. Community dental health. 1995;12(3):143-50.

17. Ferreira R, Murray J. Spielberger's State-Trait Anxiety Inventory: Measuring Anxiety with and without an Audience during Performance on a Stabilometer. Perceptual and Motor Skills. 1983;57(1):15-8.

18. Newton JT, Buck DJ. Anxiety and pain measures in dentistry: a guide to their quality and application. Journal of the American Dental Association (1939). 2000;131(10):1449-57.

19. Yuenyongchaiwat K, Baker IS, Sheffield D. Symptoms of anxiety and depression are related to cardiovascular responses to active, but not passive, coping tasks. Revista brasileira de psiquiatria (Sao Paulo, Brazil : 1999). 2017;39(2):110-7.

20. Pointer MA, Yancey S, Abou-Chacra R, Petrusi P, Waters SJ, McClelland MK. State Anxiety Is Associated with Cardiovascular Reactivity in Young, Healthy African Americans. International Journal of Hypertension. 2012;2012:7.

21. Montebugnoli L, Servidio D, Miaton RA, Prati C. Heart rate variability: a sensitive parameter for detecting abnormal cardiocirculatory changes during a stressful dental procedure. Journal of the American Dental Association (1939). 2004;135(12):1718-23.

22. Kaur R, Jindal R, Dua R, Mahajan S, Sethi K, Garg S. Comparative evaluation of the effectiveness of audio and audiovisual distraction aids in the management of anxious pediatric dental patients. Journal of the Indian Society of Pedodontics and Preventive Dentistry. 2015;33(3):192203. 
23. Navit S, Johri N, Khan SA, Singh RK, Chadha D, Navit $\mathrm{P}$, et al. Effectiveness and Comparison of Various Audio Distraction Aids in Management of Anxious Dental Paediatric Patients. Journal of clinical and diagnostic research : JCDR. 2015;9(12):Zc05-9.

24. Jindal R, Kaur R. Can We Tune Our Pediatric Patients? International Journal of Clinical Pediatric Dentistry. 2011;4(3):186-9.

25. Marwah N, Prabhakar AR, Raju OS. Music distraction-its efficacy in management of anxious pediatric dental patients. Journal of the Indian Society of Pedodontics and Preventive Dentistry. 2005;23(4):168-70.
26. Song M, Li N, Zhang X, Shang Y, Yan L, Chu J, et al. Music for reducing the anxiety and pain of patients undergoing a biopsy: A meta-analysis. Journal of advanced nursing. 2017.

27. Rezvan A, Amaya S, Betson L, Yazdany T. Randomized controlled trial of the effect of environment on patient embarrassment and anxiety with urodynamics. International urogynecology journal. 2018;29(2):291-6.

28. Zhang C, Qin D, Shen L, Ji P, Wang J. Does audiovisual distraction reduce dental anxiety in children under local anesthesia? A systematic review and meta-analysis. Oral diseases. 2018 